\title{
Characterisation and outcomes of adult patients with paracetamol overdose presenting to a tertiary hospital in Singapore
}

\author{
Christina Jiun-Yu $\underline{\operatorname{Tan}}^{1}$, PharmD, Grant E Sklar ${ }^{2,3}$, PharmD, BCPS
}

INTRODUCTION Paracetamol is the most common pharmaceutical agent implicated in toxic exposure in Singapore. This study aimed to describe the characteristics of paracetamol overdose in the adult population managed at a tertiary healthcare facility in Singapore.

METHODS Medical records of adult patients hospitalised with a diagnosis of paracetamol overdose at National University Hospital, Singapore, over a three-year period from January 2011 to December 2013 were retrospectively reviewed.

RESULTS A total of 177 patients had paracetamol overdose. The median age was 25 years, with a significant female predominance $(71.2 \%)$. Intentional ingestion accounted for the majority (76.8\%) of cases. The median dose of paracetamol ingested was 10 (interquartile range 8-15) g. Among patients who reported ingesting more than $10 \mathrm{~g}, 46.5 \%$ perceived the overdose as non-lethal. $\mathrm{N}$-acetylcysteine was administered in $76.3 \%$ of patients, among whom $24.4 \%$ experienced an anaphylactoid reaction. Of the 10 (5.6\%) patients who had severe hepatotoxicity, 2 (1.1\%) developed acute liver failure. Most patients had resolving transaminases at discharge and none required liver transplantation. The median length of hospitalisation was three days. There were no fatalities.

CONCLUSION Paracetamol overdose occurred predominantly in young adults with intentional ingestion, suggesting that preventive measures targeted at promoting public awareness may not suffice. However, the perceived lack of lethality by many patients who ingested potentially toxic amounts of paracetamol reflects a certain knowledge gap. Healthcare providers should proactively educate consumers on the proper use of paracetamol and the consequences of its overdose.

Keywords: acetaminophen, adult, drug overdose, liver failure, paracetamol

\section{INTRODUCTION}

Paracetamol is widely available in Singapore without restrictions on prescribing or dispensing. ${ }^{(1)}$ Despite its safety when used within recommended doses, paracetamol overdose has the potential to cause hepatotoxicity, the severity of which ranges from asymptomatic or mild transaminitis to acute liver failure (ALF) and even death. ${ }^{(2)}$ Paracetamol overdose remains one of the most common causes of poisoning worldwide, and is the leading cause of ALF in the United States (US) and United Kingdom (UK). ${ }^{(3,4)}$

In previous retrospective studies conducted in Singapore, paracetamol was identified to be the most common pharmaceutical agent implicated in toxic exposure and self-poisoning, accounting for $55 \%$ of suicide overdose attempts among youths below 21 years of age..$^{(5,6)}$ Specifically among the paediatric population, outcomes of paracetamol overdose appeared to be favourable, with no significant morbidity or mortality, as observed in a case series. ${ }^{(7)}$ However, given that the majority of patients in that study were aged five years or younger, no inference can be drawn with regard to the profile of paracetamol overdose cases in the local adult population. ${ }^{(7)}$ Several descriptive studies conducted in the Asian region reported generally low rates of fulminant hepatic failure and mortality associated with paracetamol overdose in adults, ${ }^{(8-10)}$ in contrast to the high healthcare burden seen in Western countries. ${ }^{(3-4)}$ Outcomes associated with paracetamol overdose in the Singapore adult population remain unclear. Hence, the objective of this study was to describe the characteristics of paracetamol overdose among the local adult patient population in terms of demographics, circumstances of overdose, management and outcomes. An improved understanding of the patterns of paracetamol overdose and its management may potentially help identify strategies to reduce the rates of paracetamol overdose, improve treatment outcomes and minimise adverse effects associated with $\mathrm{N}$-acetylcysteine (NAC) treatment.

\section{METHODS}

Medical records of adult patients admitted for paracetamol overdose to National University Hospital (NUH), Singapore, over a three-year period from 1 January 2011 to 31 December 2013 were retrospectively reviewed. Ethics committee approval was obtained from the National Healthcare Group Domain Specific Review Board prior to the commencement of the study. Case visits with a discharge diagnosis matching codes 965.4 (poisoning by aromatic analgesics, not elsewhere classified) according to the International Classification of Disease, Ninth Revision, Clinical Modification (ICD-9-CM), (11) or T39.1 (poisoning by non-opioid analgesics, antipyretics and antirheumatics [4-aminophenol derivatives]) according to the International Statistical Classification of Diseases and Related Health Problems, Tenth Revision (ICD-10), ${ }^{(12)}$ were identified from the electronic

${ }^{1}$ Department of Pharmacy, Tan Tock Seng Hospital, ${ }^{2}$ Department of Pharmacy, National University of Singapore, ${ }^{3}$ Department of Pharmacy, National University Hospital, Singapore Correspondence: Dr Grant E Sklar, Associate Professor, Department of Pharmacy, National University of Singapore, Block S4A, 18 Science Drive 4, Singapore 117543. phages@nus.edu.sg 
patient administration system. Only patients aged $\geq 18$ years were included in the study.

Clinical information of each identified patient was collected through a detailed review of medical records. Patients who had matching ICD codes but presented with a history of a single ingestion of $<2 \mathrm{~g}$ paracetamol, ingestion of $<4 \mathrm{~g}$ paracetamol over a 24-hour period, or no evidence of paracetamol ingestion were excluded. Patients who refused blood-taking were also excluded. Information extracted from the medical records included demographic data (e.g. age, gender, ethnicity, history of alcohol use and pertinent comorbidities), circumstances of overdose (e.g. intentionality of overdose, alleged dose of paracetamol ingested based on patient's recall, time course of ingestion and concomitant ingestion of other drugs), clinical features of overdose, laboratory data (e.g. plasma paracetamol level, liver function tests, renal profile, coagulation profile, serum bicarbonate and blood $\mathrm{pH}$ levels), treatment details (e.g. time to presentation at the emergency department, time to administration of NAC, activated charcoal or gastric lavage, and adverse effects from NAC treatment) and clinical outcomes. Where available, the patient's perceived lethality of the overdose attempt was also recorded.

Acute overdose was defined as ingestion of supratherapeutic doses over a period $\leq 2$ hours. A two-hour time frame was selected because it has been observed that many single overdoses were ingested over a time period of up to two hours, ${ }^{(13)}$ and this time frame is reasonably narrow for the Rumack-Matthew nomogram to be used to predict the risk of hepatotoxicity based on timed plasma paracetamol levels. Supratherapeutic doses ingested over a time period $>2$ hours were considered as staggered overdose or repeated supratherapeutic ingestion, which was collectively referred to as 'staggered ingestion' in this study.

Unintentional overdose was defined as ingestion of supratherapeutic doses for therapeutic intent without deliberate self-harm. For patients presenting with an acute overdose where plasma paracetamol levels were obtained at $\geq 4$ hours and $<24$ hours post-ingestion, the risk of hepatotoxicity was predicted using the Rumack-Matthew nomogram and classified into three risk categories. Where ingestion occurred over a time frame of $\leq 2$ hours, the earliest time point was used as the time of ingestion. Patients with plasma paracetamol levels above the 200-line were considered to be at 'probable risk'; patients with plasma paracetamol levels below the 150-line were considered to be at 'no risk'; and patients with plasma paracetamol levels falling between the 150- and 200-line were considered to be at 'possible risk' of hepatotoxicity. ${ }^{(14)}$

Paracetamol-induced hepatotoxicity was defined as hepatic dysfunction in the presence of a history consistent with excessive paracetamol ingestion and when alternative causes of acute hepatic dysfunction were less likely. Hepatocellular injury was defined as an alanine aminotransferase (ALT) level $>3$ times the upper normal limit. ${ }^{(15)}$ Severe hepatotoxicity was defined as a peak aspartate aminotransferase level > 1,000 IU/L. ${ }^{(16)}$ Patients were defined as experiencing ALF when they met the criteria of severe hepatotoxicity, coagulopathy (international normalised ratio $[I N R] \geq 1.5$ ) and any degree of encephalopathy. This criterion is consistent with the widely accepted definition of ALF and has been utilised in other retrospective studies. ${ }^{(17,18)}$

Demographic data and clinical values were presented as median and interquartile ranges (IQR) or percentage values unless otherwise specified. Between-group comparisons were made using Fisher's exact or chi-square tests for categorical variables, and Mann-Whitney $U$ test for continuous variables. Correlation analyses were made using Spearman's rank correlation coefficient. Univariate logistic regression analysis was used to identify factors associated with the development of hepatotoxicity. Statistical analysis was performed using IBM SPSS Statistics version 21.0 (IBM Corp, Armonk, NY, USA). A two-tailed $p<0.05$ was considered to be statistically significant.

\section{RESULTS}

Over the three-year period, 237 patients were discharged from NUH with diagnoses matching the specified ICD codes. However, 60 patients were excluded from the analysis, as they were under 18 years of age $(n=54)$ or refused blood-taking $(n=1)$, and because a paracetamol overdose was considered unlikely based on documented history and undetectable plasma paracetamol levels $(n=5)$. The remaining 177 patient visits were included in the study. Baseline demographics and clinical characteristics of the overall study cohort are shown in Table I.

The median age of adults admitted with paracetamol overdose was 25 (range 18-75) years. The distribution of patients by age is shown in Table II. Half of these patients ( $n=89,50.3 \%$ ) were in the 18-25-year age group, with a decreasing number of patients in the older age groups; this trend was particularly evident among patients with intentional overdose. There was a clear predominance of women in the overall cohort and among patients with intentional overdose $(75.0 \%$ of women in intentional overdose vs. $57.5 \%$ in unintentional overdose). The majority of patients were of Chinese (59.3\%) ethnicity, with similar proportions of Malay and Indian patients (14.7\% each). Compared to Singapore's national demographic distribution in 2012, our cohort had proportionately more patients of Indian and other ethnicities than those of Chinese ethnicity. ${ }^{(19}$

The median dose of paracetamol consumed was 10 (IQR 8-15) g, with $79.7 \%$ of patients ingesting a dose $\geq 7.5 \mathrm{~g}$. The doses of paracetamol ingested were similar in cases of acute and staggered overdose $(p=0.38)$. However, a greater amount of paracetamol was consumed among patients with intentional overdose $(p=0.01)$. Mixed ingestions were common $(54.8 \%)$, with caffeine $(20.3 \%)$ and alcohol $(19.2 \%)$ being the most frequent co-ingestants. Nausea and/or vomiting (83.1\%) were the most frequent symptoms of overdose observed. Of the 14 (7.9\%) patients who developed coagulopathy (defined as INR $\geq 1.5$ ), two patients did not exhibit elevated transaminases and one patient had only mild, transient transaminitis; the remaining 11 patients had evidence of hepatocellular injury. Bleeding events were noted in $12(6.8 \%)$ patients; of these, 11 patients had blood in their vomitus and one patient experienced bruising at an injection site. Only three of these 12 patients had INR $\geq 1.5$. 
Table I. Baseline demographics and clinical characteristics of study cohort $(n=177)$.

\begin{tabular}{|c|c|c|c|}
\hline Variable & No. (\%)/median (IQR) & Variable & No. (\%)/median (IQR) \\
\hline Age (yr) & $25(21-36)$ & Dose of paracetamol ingested (g) & \\
\hline Female gender & $126(71.2)$ & By time course of ingestion & \\
\hline Ethnicity & & Acute ingestion & $10(8-15)$ \\
\hline Chinese & $105(59.3)$ & Staggered ingestion & $10(7.5-10)$ \\
\hline Malay & $26(14.7)$ & By intentionality & \\
\hline Indian & $26(14.7)$ & Intentional overdose & $10(8-15)$ \\
\hline Others & $20(11.3)$ & Unintentional overdose & $9.8(7.5-10)$ \\
\hline History of alcohol use* & & Co-ingestants ${ }^{*}$ & \\
\hline Chronic drinker & $16(9.0)$ & None & $78(44.1)$ \\
\hline Social drinker & $53(29.9)$ & Caffeine & $36(20.3)$ \\
\hline None/rarely & $100(56.5)$ & Alcohol & $34(19.2)$ \\
\hline Missing data & $8(4.5)$ & Orphenadrine & $15(8.5)$ \\
\hline Circumstance of overdose & & Clinical features of overdose & \\
\hline Intentionality of overdose & & None/asymptomatic & $3(1.7)$ \\
\hline Intentional & $136(76.8)$ & Nausea/vomiting & $147(83.1)$ \\
\hline Acute $^{+}$ & 126 & Abdominal pain & $96(54.2)$ \\
\hline Staggered ${ }^{+}$ & 9 & Dizziness & $95(53.7)$ \\
\hline Unknown ${ }^{+}$ & 1 & Hypokalaemia & $69(39.0)$ \\
\hline Unintentional & $40(22.6)$ & Drowsiness & $54(30.5)$ \\
\hline Acute $^{+}$ & 28 & Coagulopathy (INR $\geq 1.5$ ) & $14(7.9)$ \\
\hline Staggered $^{+}$ & 12 & Bleeding & $12(6.8)$ \\
\hline Intent unclear & $1(0.6)$ & Confusion & $8(4.5)$ \\
\hline Time course of ingestion & & Jaundice & $4(2.3)$ \\
\hline Acute ingestion & $154(87.0)$ & Treatment instituted & \\
\hline Staggered ingestion & $21(11.9)$ & $\mathrm{N}$-acetylcysteine & $135(76.3)$ \\
\hline$\leq 8 h$ & $15(8.5)$ & General supportive care only & $38(21.5)$ \\
\hline$>8 h$ & $6(3.4)$ & Activated charcoal & $27(15.3)$ \\
\hline Unknown & $2(1.1)$ & Gastric lavage & $3(1.7)$ \\
\hline
\end{tabular}

*History of alcohol use was recorded as chronic drinker, social drinker or non-drinker based on documentation of alcohol use in the medical records. †Acute overdose: ingestion of supratherapeutic doses over a period $\leq 2 \mathrm{~h}$; staggered overdose: supratherapeutic doses ingested over a time period $>2 \mathrm{~h}$; unknown: time course of ingestion was unclear based on documented history. $¥$ Co-ingestants listed were not exhaustive or mutually exclusive; $23.7 \%$ of cases reported more than one co-ingestant; presence of co-ingestant in $1.1 \%$ of patients was unclear. INR: international normalised ratio; IQR: interquartile range

Table II. Age distribution of patients stratified by intentionality of overdose $(n=177)$.

\begin{tabular}{lccc}
\hline Age group (yr) & \multicolumn{3}{c}{ No. of patients } \\
\cline { 2 - 4 } & Intentional & Unintentional & Unclear \\
\hline $18-25$ & 65 & 23 & 1 \\
$26-35$ & 37 & 4 & - \\
$36-45$ & 18 & 8 & - \\
$46-55$ & 10 & 4 & - \\
$\geq 56$ & 6 & 1 & - \\
\hline
\end{tabular}

Treatment using gastric lavage was instituted for $3(1.7 \%)$ patients who had ingested $>300 \mathrm{mg} / \mathrm{kg}$ of paracetamol and presented to the hospital within 1-4 hours of acute ingestion. NAC was initiated in 135 (76.3\%) patients, and 112 of these patients completed the full course of intravenous NAC regimen (given as an initial dose of $150 \mathrm{mg} / \mathrm{kg}$ over 15-60 minutes, followed by $50 \mathrm{mg} / \mathrm{kg}$ over four hours and then $100 \mathrm{mg} / \mathrm{kg}$ over 16 hours). The characteristics of patients treated with NAC are summarised in Table III. The median time from presentation at the emergency department to receipt of NAC treatment was 1.4 (IQR 0.9-2.4) hours. Among the study cohort, 42 patients did not receive NAC: 27 patients had plasma paracetamol levels indicating no risk of toxicity; five were assessed by the managing physician to have low likelihood of toxicity based on plasma paracetamol levels or history; and ten had no clear reason documented. One of the latter patients was not initiated on NAC despite showing signs of transaminitis on presentation to the emergency department.

Most $(73.3 \%)$ of the patients who were initiated on NAC tolerated the treatment without adverse effects. Anaphylactoid reaction, a composite of symptoms that include flushing, redness, urticaria, shortness of breath and/or angioedema, occurred in $33(24.4 \%)$ patients. Of these 33 patients, 13 patients had the intravenous NAC treatment terminated, among whom two were switched to alternatives (one was switched to oral NAC while the other to methionine), while the rate of infusion was reduced in 12 patients, among whom seven patients were also prescribed an antihistamine and/or hydrocortisone. Two patients were prescribed an antihistamine and/or hydrocortisone without 
Table III. Characteristics of patients treated with NAC $(n=135)$.

\begin{tabular}{lc}
\hline Variable & No. (\%) \\
\hline Time course of ingestion* & $117(86.7)$ \\
Acute & $18(13.3)$ \\
Staggered/unknown & \\
NAC regimen & $112(83.7)$ \\
$\quad$ Full course administered & $23(16.3)$ \\
Regimen terminated before full course was & \\
completed & \\
Overall cohort & $1.4(0.9-2.4)$ \\
Time from ED presentation to NAC & \\
administration (hr) & \\
Adverse effects to NAC* & \\
None & $99(73.3)$ \\
Gastrointestinal (e.g. nausea/vomiting) & $53(3.7)$ \\
Anaphylactoid reaction & $33.4)$ \\
\hline
\end{tabular}

*Acute overdose: ingestion of supratherapeutic doses over a period $\leq 2 \mathrm{hr}$ staggered overdose: ingestion of supratherapeutic doses over a period $>2 \mathrm{hr}$; unknown: time course of ingestion was unclear based on documented history. †Data presented as median (interquartile range). ${ }^{*}$ Patients may have $>1$ adverse effect. ED: emergency department; NAC: N-acetylcysteine

slowing the rate of infusion of intravenous NAC. There were no documented interventions for six patients. Among the 33 patients with anaphylactoid reactions, 11 patients received treatment with an antihistamine, two patients received treatment with hydrocortisone and five patients received both. The incidence of anaphylactoid reactions among patients with or without a history of asthma was comparable (29.4\% vs. $23.7 \%$, respectively; $\mathrm{p}=0.56)$.

The perceived lethality of the overdose attempt was prospectively surveyed during psychiatric consultation using a standardised suicide risk assessment form, which was part of standard care. Although 167 patients had at least one psychiatric review, this information was only available for 114 patients. Among the patients who ingested $\geq 10 \mathrm{~g}$ of paracetamol, $46.5 \%$ of them perceived the overdose as non-lethal, $45.1 \%$ perceived it as moderately or very lethal and the remaining patients were not sure. A comparison of patients who perceived the overdose as non-lethal with those who perceived it as moderately or very lethal showed that there were more men $(33.3 \%$ vs. $18.8 \%$, respectively) and unintentional overdoses (33.3\% vs. $3.1 \%$, respectively) in the former group. The most common reason for unintentional overdose was treatment of pain, with headache $(57.9 \%)$ being the most common type of pain reported.

A total of 154 patients presented with acute ingestions, one of whom was unclear about the time of ingestion. There was a positive but weak correlation between the risk of hepatotoxicity predicted using the Rumack-Matthew nomogram and the alleged amount of paracetamol ingested, for both the absolute quantity and weight-based $(\mathrm{mg} / \mathrm{kg})$ quantity $(\mathrm{r}=0.37$ and 0.44 , respectively; $p<0.01$ ), as shown in Table IV. Among patients with a known estimated time of ingestion, the median time from ingestion to hospital presentation was 4.2 (IQR 2.0-9.4) hours. Most patients presented within the first seven hours of ingestion $(63.4 \%)$, providing opportunity for NAC initiation within the first
Table IV. Characteristics of patients presenting with acute overdose $(n=154)$.

\begin{tabular}{|c|c|}
\hline Variable & Median (IQR) \\
\hline \multicolumn{2}{|c|}{$\begin{array}{l}\text { Dose of paracetamol ingested stratified by } \\
\text { risk of hepatotoxicity }(\mathrm{g})^{*}\end{array}$} \\
\hline Probable risk & $15.0(10.0-17.3)$ \\
\hline Possible risk & $11.3(8.0-15.0)$ \\
\hline No risk & $9.0(6.0-10.0)$ \\
\hline \multicolumn{2}{|c|}{$\begin{array}{l}\text { Weight-based dose of paracetamol ingested } \\
\text { stratified by risk of hepatotoxicity }(\mathrm{mg} / \mathrm{kg})^{*}\end{array}$} \\
\hline Probable risk & $252(203-325)$ \\
\hline Possible risk & $176(139-261)$ \\
\hline No risk & 149 (117-205) \\
\hline \multicolumn{2}{|c|}{ Time interval from ingestion ${ }^{+}(\mathrm{hr})(\mathrm{n}=153)$} \\
\hline To hospital presentation & $4.2(2.0-9.4)$ \\
\hline To treatment with NAC $(n=116)^{\ddagger}$ & $6.1(3.5-11.7)$ \\
\hline $\begin{array}{l}\text { To treatment with activated charcoal } \\
(n=26)\end{array}$ & $3.9(2.9-5.7)$ \\
\hline
\end{tabular}

${ }^{*}$ Risk of hepatotoxicity as predicted using the Rumack-Matthew nomogram. †Among patients with known time of ingestion. $¥ 117$ patients with acute overdose received NAC but one patient was excluded from the analysis, as time of ingestion was unknown. IQR: interquartile range; NAC: N-acetylcysteine

eight hours. Only 5 (3.2\%) patients presented more than 24 hours after ingestion.

Activated charcoal was administered in 26 patients with acute overdose, and the median time from ingestion to treatment was 3.9 (IQR 2.9-5.7) hours. Three patients received activated charcoal more than ten hours after the estimated time of ingestion. NAC was administered in 117 (76.0\%) patients with acute overdose. Among the 61 patients who were indicated for NAC treatment (i.e. predicted probable risk or possible risk based on the nomogram), $39.3 \%$ and $77.0 \%$ of patients received NAC within the first eight and first 16 hours of overdose, respectively. The most common reason for delay in NAC administration beyond eight hours of overdose was late presentation to the emergency department (79.6\%). Logistics delay, defined collectively as delay due to pending paracetamol levels before initiation of treatment or delay from the time of medication order to its administration, accounted for $12.2 \%$ of delays in NAC administration. Despite a predicted probable or possible risk of hepatotoxicity based on timed plasma paracetamol levels, $5(8.2 \%)$ patients were not initiated on NAC treatment and $6(9.8 \%)$ patients had the NAC regimen prematurely terminated without the use of alternative treatment. Reasons for early discontinuation of treatment included development of adverse effects to NAC $(n=2)$, patient discharged against medical advice $(n=1)$ and discontinuation of treatment upon transfer from the emergency department to the ward with no documented reason $(n=3)$. None of these patients developed hepatotoxicity. Of the patients with plasma paracetamol levels that predicted no risk of hepatotoxicity, 33 (45.2\%) patients were prescribed a full course of NAC. One of these patients had elevated lactate and low serum bicarbonate levels despite nontoxic plasma paracetamol levels. Nine patients were treated with a complete regimen of NAC based on plasma paracetamol levels taken within the first four hours of ingestion. 
Clinical outcomes of patients in the study cohort are presented in Table $\mathrm{V}$. The median length of hospitalisation was 3 (range 1-28) days. Three patients required intensive care unit (ICU) admission, with a length of stay of 2-4 days. Of these patients, two were admitted to the ICU for severe metabolic acidosis. Both patients had ingested approximately $300 \mathrm{mg} / \mathrm{kg}$ of paracetamol, had a plasma paracetamol level of 120-125 mg/L at approximately five hours post-ingestion, received prompt treatment with NAC within three hours of ingestion, and did not develop hepatocellular injury. One of these patients reported co-ingestion of orphenadrine, chlorpheniramine and bromhexine; the other had suspected co-ingestion of risperidone based on the obtained circumstantial history, but toxicology screen was negative for risperidone and positive for lignocaine and pseudoephedrine only. The third patient was admitted to the ICU for severe hypotension caused by co-ingested antihypertensive agents.

A total of $16(9.0 \%)$ patients developed hepatocellular injury. Of these, one patient had plasma paracetamol levels that predicted 'no risk' according to the nomogram. This patient, who had no obvious predisposing risk factors (i.e. the patient was young, non-alcoholic and with no significant comorbidities), reported a single ingestion of $7.5 \mathrm{~g}$ of paracetamol for severe headache seven hours prior to presentation, and denied any co-ingestant. He did not receive NAC treatment and developed a peak ALT level of 222 IU/L. 10 (5.6\%) patients developed severe hepatotoxicity, among whom two patients fulfilled the criteria of ALF and two other patients had concomitant acute renal impairment. One of these ten patients, a 36-year-old man with no apparent risk factors for hepatotoxicity (i.e. he was a social drinker and had no prior liver disease), had been transferred from another institution for further management of severe hepatotoxicity, in case a liver transplantation was required. He had reportedly ingested an estimated quantity of $7.5 \mathrm{~g}$ of paracetamol over a seven-hour period for migraine and presented to a medical facility with vomiting, diarrhoea, abdominal pain and fever more than two days after the ingestion. On admission, his plasma paracetamol and transaminase levels were $10 \mathrm{mg} / \mathrm{L}$ and > 4,000 IU/L, respectively. He was diagnosed with sepsis from suspected gastroenteritis, and acute hepatitis that was suspected to be associated with paracetamol. Table VI describes the characteristics of patients who developed severe hepatotoxicity. Most of these patients had resolving liver enzymes at the time of discharge. None of the patients required liver transplantation. There were no fatalities among the patients included in the study.

In the overall study cohort, the dose of paracetamol ingested was predictive of hepatocellular injury and severe hepatotoxicity (odds ratio [OR] 1.07, p < 0.05). In the subgroup of patients who presented with a history of a known time of acute ingestion, increasing time delay to NAC administration was associated with higher rates of hepatocellular injury (OR 1.17, $\mathrm{p}<0.01)$ and severe hepatotoxicity $(\mathrm{OR} 1.12, \mathrm{p}=$ 0.04). Hepatocellular injury developed in half of the patients who received NAC more than 24 hours after ingestion, as compared to no instances of hepatotoxicity among patients who received NAC within the first eight hours of ingestion. There was no significant association between the occurrence of hepatocellular injury and age, intentionality of overdose, time course of ingestion, history of alcohol use or involvement of alcohol as a co-ingestant.

Table V. Clinical outcomes of study cohort $(n=177)$.

\begin{tabular}{lc}
\hline Outcome & No. (\%) \\
\hline Length of hospitalisation (day)* $^{*}$ & $3(2-5)$ \\
Hepatocellular injury $^{+}(\mathrm{ALT}>3 \times$ ULN) & $16(9.0)$ \\
Severe hepatotoxicity (AST $>1,000$ IU/L) & $10(5.6)$ \\
Acute liver failure & $2(1.1)$ \\
\hline
\end{tabular}

*Data presented as median (interquartile range). †Among 16 patients who fulfilled the criteria for hepatocellular injury, ten met the criteria for severe hepatotoxicity. ALT: alanine aminotransferase; AST: aspartate aminotransferase; ULN: upper limit of normal

Table VI. Characteristics of patients who developed severe hepatotoxicity following paracetamol overdose $(\mathrm{n}=10)$.

\begin{tabular}{|c|c|c|c|c|c|c|c|c|}
\hline Age (yr)/gender & Dose (g) & Dose (mg/kg) & Intentionality & $\begin{array}{l}\text { Time course } \\
\text { of ingestion }\end{array}$ & $\begin{array}{l}\text { Time to ED } \\
\text { visit* (hr) }\end{array}$ & $\begin{array}{l}\text { Predicted } \\
\text { risk }^{+}\end{array}$ & $\begin{array}{l}\text { Time to } \\
\text { NAC (hr) }\end{array}$ & ALF \\
\hline $64 / \mathrm{male}$ & 35 & 540 & Yes & Acute & 8.0 & Probable & 8.9 & Yes \\
\hline 41/female & 25 & 500 & Yes & Acute & 18.7 & Probable & 20.2 & No \\
\hline $45 /$ female & 25 & 452 & Yes & Acute & 136.6 & NA & 146.8 & No \\
\hline 18/female & 15 & 300 & Yes & Acute & 7.2 & Probable & 9.7 & No \\
\hline 23/female & 14 & 245 & Yes & Staggered (> $5 \mathrm{hr}$ ) & $\sim 9.0$ & NA & 10.0 & No \\
\hline $27 /$ female & 8 & 133 & Yes & Acute & 5.4 & Possible & $18.6^{\ddagger}$ & No \\
\hline $36 / \mathrm{male}$ & 7.5 & 104 & No & Staggered (> $7 \mathrm{hr}$ ) & $\sim 55.0$ & NA & $\sim 57.0$ & No \\
\hline 24/female & - & - & - & - & - & NA & - & No \\
\hline
\end{tabular}

All ten patients who developed severe hepatotoxicity received treatment with NAC. *For staggered overdose, time to ED visit was estimated from the time of first ingestion. +Refers to the risk of hepatotoxicity predicted using the Rumack-Matthew nomogram with timed plasma paracetamol levels obtained after at least 4 hr and before $24 \mathrm{hr}$ post-ingestion. Predicted risk of hepatotoxicity was not applicable for patients with plasma paracetamol levels obtained over 24 hr post-ingestion or with history of staggered overdose. ¥NAC administration was delayed due to the transfer of patients from the ED to the general ward and from the time the medication was ordered to its administration. §Details of overdose (including dose, time of ingestion and intentionality) were not known for this patient. ALF: acute liver failure; ED: emergency department; NA: not applicable; NAC: N-acetylcysteine 


\section{DISCUSSION}

In keeping with studies conducted in other regions, such as in the US, ${ }^{(3)}$ Australia, ${ }^{(20)}$ Malaysia $^{(8)}$ and Hong Kong, ${ }^{(10)}$ patients with paracetamol overdose in our study cohort were predominantly women, with significant clustering in the age group of 18-35 years. The ethnic distribution in our cohort closely mirrors the distribution observed in a retrospective evaluation of toxic exposures conducted in a local setting ten years ago. ${ }^{(5)}$ The majority of paracetamol overdose cases were intentional and involved acute ingestion, suggesting that paracetamol overdose would be difficult to prevent with measures solely targeted at enhancing public awareness. However, the perceived lack of lethality in almost half of our patients who ingested a potentially toxic dose of paracetamol reflects a certain knowledge gap within the local population.

Although there are 116 paracetamol-containing products registered in Singapore, ${ }^{(21)}$ none of the unintentional overdoses in this study involved the use of more than one paracetamol product. Most of the unintentional overdoses involved patients unknowingly taking more than the labelled dose to seek additional therapeutic benefit. These findings suggest that healthcare providers may potentially reduce these avoidable overdose occurrences and admissions through proactive education of consumers on the proper use of paracetamol, the potential consequences of overdose, and the availability of alternative analgesics when paracetamol provides inadequate analgesia.

$\mathrm{NUH}$ is one of the two public institutions in Singapore that provides a liver transplant service. It was expected that some of the patients who received initial treatment elsewhere but developed fulminant hepatic failure would be referred to our institution for further management should transplant be considered necessary. Despite this, ALF rarely occurred and there were no fatalities observed, which was consistent with the low rate of mortality observed from Hong Kong ${ }^{(10)}$ and Malaysia. ${ }^{(8)}$ This may be explained by the low prevalence of chronic alcohol use and early administration of NAC following an overdose, as observed in this study.

Schmidt et al previously identified chronic alcohol use, quantity of paracetamol ingested and delayed time to NAC treatment as positive predictors of paracetamol-induced hepatotoxicity. ${ }^{(22)}$ In our study, chronic alcohol use $(9.0 \%)$ was uncommon. However, most patients presented with a history of significant ingestion of paracetamol. A potentially toxic threshold dose of paracetamol has not been clearly determined. Based on a consensus guideline for out-of-hospital management of paracetamol overdose, evaluation at a medical facility is recommended if: (a) at least $10 \mathrm{~g}$ or $200 \mathrm{mg} / \mathrm{kg}$ (whichever is less) is ingested within an eight-hour period for single ingestion; (b) in the case of repeated supratherapeutic ingestion, at least $10 \mathrm{~g}$ or $200 \mathrm{mg} / \mathrm{kg}$ (whichever is less) is ingested within a 24-hour period; or (c) at least $6 \mathrm{~g}$ or 150 $\mathrm{mg} / \mathrm{kg}$ (whichever is less) is ingested per 24-hour period for 48 hours or longer. ${ }^{(23)}$ Bernal cited $7.5 \mathrm{~g}$ or $125 \mathrm{mg} / \mathrm{kg}$ as the minimum dose capable of causing hepatotoxicity. ${ }^{(24)}$ Among the patients who developed severe hepatotoxicity in our study, the majority had ingested at least $10 \mathrm{~g}$ or $200 \mathrm{mg} / \mathrm{kg}$ within an eight-hour period. Two patients developed severe hepatotoxicity despite ingesting lower doses; the postulated reasons include inaccurate patient recall of ingested doses, delayed NAC treatment and/or increased susceptibility to paracetamol poisoning at lower doses due to genetic polymorphisms. ${ }^{(25)}$

Time to receipt of NAC treatment has been well established as a major determinant of paracetamol-induced hepatotoxicity. Early NAC treatment within eight hours of ingestion is highly effective in preventing serious hepatotoxicity, as seen in our study and previous studies. ${ }^{(26,27)}$ Time delay beyond the eighthour window period is associated with a progressive increase in risk of hepatotoxicity. In a multicentre study conducted in the US, the rates of severe hepatotoxicity among patients with plasma paracetamol concentrations in the probable risk zones were $6.1 \%, 20.1 \%$ and $41.0 \%$ when time to NAC treatment was $<10$ hours, 10-16 hours and 16-24 hours, respectively. ${ }^{(27)}$ In our study, the majority of patients presented early to the facility, allowing for NAC administration within 16 hours of ingestion. NAC was often initiated based on the reported dose ingested and prior to the availability of plasma paracetamol levels to maximise the potential benefit of early NAC use, which is consistent with local published guidance. ${ }^{(28)}$ However, NAC was also continued in almost half of our patients in whom plasma paracetamol levels indicated no risk of hepatotoxicity. Continued administration of NAC to complete a full course is a reasonable approach if the time of paracetamol ingestion is uncertain and the patient has a measurable paracetamol level, or if the patient is exhibiting hepatotoxicity suspected to be attributed to paracetamol. ${ }^{(29-31)}$ However, indiscriminate use of NAC should be avoided, as NAC treatment is not without risks.

The main concerns with NAC use include gastrointestinal side effects and anaphylactoid reactions. The rates of gastrointestinal side effects associated with NAC reported in the literature are widely variable, ranging from $3 \%$ to $70 \%$. ${ }^{(32)}$ Nausea and/or vomiting attributable to NAC administration was observed in less than $4 \%$ of patients receiving NAC in our study. However, this may be an underestimation, as nausea and/or vomiting were presenting symptoms of paracetamol overdose in most patients. As a result, it is likely that the continued symptoms could not be differentiated from the side effects of NAC use. A number of studies and case reports have raised concerns that patients with a history of asthma may be at an increased risk of anaphylactoid reactions, while others have suggested a lack of association. ${ }^{(33-36)}$ Carroll et al observed a three-fold higher prevalence of asthma in patients experiencing an anaphylactoid reaction to NAC, ${ }^{(33)}$ similar to the findings from a large cohort study in Denmark..$^{(35)}$ On the other hand, Schmidt found no significant association between the occurrence of NAC adverse effects and a history of asthma. ${ }^{(36)}$ Similarly, our study showed no significant excess risk of anaphylactoid reactions among patients with a history of asthma. However, this conclusion is limited by the small sample size of the current study, which was also not specifically designed to examine such an association. 
Published data has also suggested that unintentional overdose is associated with a worse outcome, likely contributed by late presentation to a medical facility and delayed initiation of treatment. ${ }^{(3,37)}$ The United States Acute Liver Failure Study Group reported that unintentional overdoses constituted $48 \%$ of paracetamol-induced ALF despite its lower representation in the overall cohort of paracetamol-associated hospitalisations. ${ }^{(3)}$ A retrospective review in Canada reported similar findings, concluding that patients with unintentional overdose were at a five-fold increased risk of hepatotoxicity. ${ }^{(37)}$ This pattern was evident in smaller studies as well. ${ }^{(38,39)}$ However, in our study, although patients with unintentional overdose constituted $22.6 \%$ of the overall cohort, only one in ten $(10 \%)$ cases of severe hepatotoxicity was caused by unintentional overdose. This disparity may be explained by the difference in the pattern of unintentional overdoses observed in our study. Although staggered ingestion was more common among patients with unintentional overdose, none of these ingestions among patients with unintentional overdose occurred over a time frame of greater than 48 hours. Several reasons could be postulated to account for the absence of patients presenting with a history of chronic supratherapeutic ingestion in our study. Firstly, this subgroup of patients may not have been captured because patients who develop liver damage from chronic ingestion of supratherapeutic doses may not associate the symptoms with paracetamol poisoning and may fail to provide clinicians with the relevant history; hence, they may not be labelled with the correct discharge diagnosis. Alternatively, such patients may be rare because patients promptly seek medical attention in an overdose scenario when they develop nonspecific symptoms, such as nausea, before significant liver damage occurs. Future studies with the intent to characterise chronic overdose should consider alternative methods to seek out such patients to determine its true incidence.

The recommended treatment threshold of paracetamol overdose with NAC in Singapore ${ }^{(28)}$ is similar to that in the US and Australia. ${ }^{(40)}$ Using the 150-line on the Rumack-Matthew nomogram, one patient was stratified as 'no risk' based on timed paracetamol level, and was not treated with NAC. However, the patient subsequently developed evidence of hepatocellular injury. None of the patients with timed plasma paracetamol levels below the 150-line developed severe hepatotoxicity or ALF. The absence of major adverse outcomes in patients stratified as 'no risk' when using the existing treatment threshold guidance suggests that there is no urgent need to adopt a more conservative threshold for treatment in the local setting.

Severe metabolic acidosis requiring ICU admission was observed in two patients in this study. Although there were suspected co-ingestants in both patients, metabolic acidosis is not a common adverse effect of the suspected co-ingested agents. Several case reports of early metabolic acidosis associated with paracetamol overdose, in the absence of hepatocellular injury, have been published in the literature. ${ }^{(41)}$ The proposed aetiology based on in vitro and in vivo studies is direct mitochondrial toxicity of paracetamol and its metabolites. ${ }^{(41)}$ However, patients in these case reports typically presented with high peak paracetamol levels (> $800 \mathrm{mg} / \mathrm{L}$ ) in contrast to the levels observed in our study. Peak paracetamol levels were not collected for these two patients, as this was not a predefined data variable in this study. Based on the available data from this retrospective review, we were unable to draw specific conclusions on the association of metabolic acidosis with supratherapeutic paracetamol levels.

Although the medical records of each patient were reviewed in detail, this retrospective observational study had several limitations. As discharge diagnosis coding was used to identify patients with paracetamol overdose in a tertiary care centre, the findings from this study may not be representative of the entire spectrum of paracetamol overdose severity in the local setting - patients who did not require admission, were managed as outpatients without hospital referral, died before reaching the hospital or were not labelled with the relevant discharge diagnoses were not captured. However, hospitalised patients likely reflected more significant paracetamol ingestion when compared to patients who were managed in out-of-hospital settings. Furthermore, it is not likely that paracetamol overdose would have resulted in sudden death. As a single-centre study, this study may not be representative of all paracetamol overdose cases in the local setting, especially since there may be inter-hospital variability in the clinical management of such patients. Being retrospective in nature, this study had missing data for several variables, such as the patient's weight and perceived lethality. In particular, detailed history of alcohol use was limited by the level of documentation in the medical records.

To conclude, in this study, the mortality rate attributable to paracetamol overdose was low, and paracetamol-induced hepatotoxicity was conservatively managed with supportive treatment and NAC, without the need for liver transplant. Most overdoses occurred in young adults with intentional ingestion. However, there appears to be a knowledge gap with regard to the potential consequences of paracetamol overdose, as reflected by the perceived lack of lethality observed in many patients with significant ingestion. Healthcare providers should proactively educate consumers on the proper use of paracetamol and the potential consequences that follow excessive ingestion. The overall low rates of severe adverse outcomes observed in this study suggest that the current treatment threshold for NAC initiation remains relevant in the local setting. Given that time to treatment is a major determinant of paracetamol overdose outcomes, utilising protocols within the emergency department for the management of these patients may help to minimise time delay to NAC treatment and improve the continuity of management during transfer of care.

\section{REFERENCES}

1. Health Sciences Authority, Singapore. HSA's Role in the Regulation of Western Medicines in Singapore. Available at: http://www.hsa.gov.sg/content/hsa/ en/Health_Products_Regulation/Consumer_Information/Consumer_Guides/ Medicine/hsa_s-role-in-the-regulation-of-western-medicines-in-singapore-.html. Accessed March 7, 2015.

2. Kux L. Federal Register Notice: Prescription Drug Products Containing Acetaminophen: Actions to Reduce Liver Injury from Unintentional Overdose. Available at: https://www.regulations.gov/document?D=FDA- 
2011-N-0021-0001. Accessed February 26, 2015.

3. Larson AM, Polson J, Fontana RJ, et al; Acute Liver Failure Study Group. Acetaminophen-induced acute liver failure: results of a United States multicenter, prospective study. Hepatology 2005; 42:1364-72.

4. Bernal W, Wendon J. Acute liver failure. N Engl J Med 2013; 369:2525-34.

5. Ponampalam R, Tan HH, Ng KC, Lee WY, Tan SC. Demographics of toxic exposures presenting to three public hospital emergency departments in Singapore 2001-2003. Int J Emerg Med 2009; 2:25-31.

6. Wai $\mathrm{BH}$, Hong $\mathrm{C}$, Heok KE. Suicidal behavior among young people in Singapore. Gen Hosp Psychiatry 1999; 21:128-33.

7. Ng KC. Paracetamol ingestions at the Children's Emergency Department--a three year series. Singapore Med J 2003; 44:79-83.

8. Marzilawati AR, Ngau YY, Mahadeva S. Low rates of hepatotoxicity among Asian patients with paracetamol overdose: a review of 1024 cases. BMC Pharmacol Toxicol 2012; 13:8.

9. Mohd Zain Z, Fathelrahman Al, Ab Rahman AF. Characteristics and outcomes of paracetamol poisoning cases at a general hospital in Northern Malaysia. Singapore Med J 2006; 47:134-7.

10. Chan TY. Fulminant hepatic failure due to acetaminophen poisoning may be less common in Hong Kong. J Toxicol Clin Toxicol 2001; 39:175-7.

11. Centers for Disease Control and Prevention. Appendix E: List of Three-Digit Categories. In: International Classification of Diseases, 9th Revision, Clinical Modification (ICD-9-CM). Available at: https://www.acro.org/washington/ International_Classification_of_Diseases.pdf. Accessed January 22, 2015.

12. World Health Organization. Chapter XIX Injury, poisoning and certain other consequences of external causes. In: International Statistical Classification of Diseases and Related Health Problems 10th Revision (ICD-10) Version for 2010 Available at: http://apps.who.int/classifications/icd10/browse/2010/en\#/T39.1. Accessed January 22, 2015.

13. Bateman DN, Dear JW, Thanacoody HK, et al. Reduction of adverse effects from intravenous acetylcysteine treatment for paracetamol poisoning: a randomised controlled trial. Lancet 2014; 383:697-704.

14. Rumack BH, Peterson RC, Koch GG, Amara IA. Acetaminophen overdose. 662 cases with evaluation of oral acetylcysteine treatment. Arch Intern Med 1981; 141:380-5.

15. Navarro VJ, Senior JR. Drug-related hepatotoxicity. N Engl J Med 2006; 354:731-9.

16. Rumack BH. Acetaminophen hepatotoxicity: the first 35 years. J Toxicol Clin Toxicol 2002; 40:3-20.

17. Polson J, Lee WM; American Association for the Study of Liver Disease. AASLD position paper: the management of acute liver failure. Hepatology 2005 41:1179-97.

18. Kjartansdottir I, Bergmann OM, Arnadottir RS, Björnsson ES. Paracetamo intoxications: a retrospective population-based study in Iceland. Scand Gastroenterol 2012; 47:1344-52.

19. Ministry of Health, Singapore. Population and Vital Statistics. Available at: https:// www.moh.gov.sg/content/moh_web/home/statistics/Health_Facts_Singapore/ Population_And_Vital_Statistics.html. Accessed January 22, 2015.

20. Ayonrinde OT, Phelps GJ, Hurley JC, Ayonrinde OA. Paracetamol overdose and hepatotoxicity at a regional Australian hospital: a 4-year experience. Intern Med J 2005; 35:655-60.

21. Health Sciences Authority, Singapore. PZ4970 Infosearch - Medicinal Products. Available at: http://eservice.hsa.gov.sg/prism/common/enquirepublic/ SearchDRBProduct.do?action=getSearchResults. Accessed August 10, 2015.

22. Schmidt LE, Dalhoff K, Poulsen HE. Acute versus chronic alcohol consumption in acetaminophen-induced hepatotoxicity. Hepatology 2002; 35:876-82.

23. Dart RC, Erdman AR, Olson KR, et al; American Association of Poison Control Centers. Acetaminophen poisoning: an evidence-based consensus guideline for out-of-hospital management. Clin Toxicol (Phila) 2006; 44:1-18.

24. Bernal W. Changing patterns of causation and the use of transplantation in the United kingdom. Semin Liver Dis 2003; 23:227-37.

25. Court MH, Duan SX, von Moltke LL, et al. Interindividual variability in acetaminophen glucuronidation by human liver microsomes: identification of relevant acetaminophen UDP-glucuronosyltransferase isoforms. J Pharmacol Exp Ther 2001; 299:998-1006.

26. Prescott LF, Illingworth RN, Critchley JA, et al. Intravenous N-acetylcystine: the treatment of choice for paracetamol poisoning. Br Med J 1979; 2:1097-100.

27. Smilkstein MJ, Knapp GL, Kulig KW, Rumack BH. Efficacy of oral $\mathrm{N}$-acetylcysteine in the treatment of acetaminophen overdose. Analysis of the national multicenter study (1976 to 1985). N Engl J Med 1988; 319:1557-62.

28. Ministry of Health, Singapore. Management of Poisoning. In: $\mathrm{MOH}$ Clinical Practice Guidelines Dec/2011. Available at: https://www.moh.gov.sg/content/ $\mathrm{dam} / \mathrm{moh}$ web/HPP/Pharmacists/cpg_pharmacy/management $\% 20$ of $\% 20$ poisoning\%20-\%20booklet.pdf. Accessed March 7, 2015.

29. Wolf SJ, Heard K, Sloan EP, Jagoda AS; American College of Emergency Physicians Clinical Policies Subcommittee (Writing Committee) on Critical Issues in the Management of Patients Presenting to the Emergency Department with Acetaminophen Overdose. Clinical policy: critical issues in the management of patients presenting to the emergency department with acetaminophen overdose. J Emerg Nurs 2008; 34:e1-18.

30. Salhanick SD, Shannon MW. Acetaminophen. In: Shannon MW, Borron SW, Burns M, eds. Haddad and Winchester's Clinical Management of Poisoning and Drug Overdose. 4th ed. Philadelphia: Saunders/Elsevier, 2007: 825-34.

31. Heard KJ. Acetylcysteine for acetaminophen poisoning. N Engl J Med 2008; 359:285-92.

32. Koppen A, van Riel A, de Vries I, Meulenbelt J. Recommendations for the paracetamol treatment nomogram and side effects of $\mathrm{N}$-acetylcysteine. Neth J Med 2014; 72:251-7.

33. Carroll R, Benger J, Bramley K, et al. Epidemiology, management and outcome of paracetamol poisoning in an inner city emergency department. Emerg Med J 2015; 32:155-60.

34. Appelboam AV, Dargan PI, Knighton J. Fatal anaphylactoid reaction to $\mathrm{N}$-acetylcysteine: caution in patients with asthma. Emerg Med J 2002; 19:594-5.

35. Schmidt LE, Dalhoff K. Risk factors in the development of adverse reactions to $\mathrm{N}$-acetylcysteine in patients with paracetamol poisoning. Br J Clin Pharmacol 2001; 51:87-91.

36. Schmidt LE. Identification of patients at risk of anaphylactoid reactions to $\mathrm{N}$-acetylcysteine in the treatment of paracetamol overdose. Clin Toxicol (Phila) 2013; 51:467-72.

37. Myers RP, Shaheen AA, Li B, Dean S, Quan H. Impact of liver disease, alcohol abuse, and unintentional ingestions on the outcomes of acetaminophen overdose. Clin Gastroenterol Hepatol 2008; 6:918-25.

38. Gyamlani GG, Parikh CR. Acetaminophen toxicity: suicidal vs. accidental. Crit Care 2002; 6:155-9.

39. Schiødt FV, Rochling FA, Casey DL, Lee WM. Acetaminophen toxicity in an urban county hospital. N Engl J Med 1997; 337:1112-7.

40. Ferner RE, Dear JW, Bateman DN. Management of paracetamol poisoning. BMJ $2011 ; 342: \mathrm{d} 2218$

41. Shah AD, Wood DM, Dargan PI. Understanding lactic acidosis in paracetamol (acetaminophen) poisoning. Br J Clin Pharmacol 2011; 71:20-8. 